\title{
Accelerometer transverse sensitivity calibration; validation and uncertainty estimation
}

\author{
Christiaan S Veldman \\ National Metrology Laboratory of South Africa, Acoustics, Ultrasound and Vibration Laboratory, South Africa Private Bag X34, Lynnwood \\ Ridge, 0004, South Africa
}

\begin{abstract}
The National Metrology Institute of South Africa (NMISA) has implemented a system to measure the transverse sensitivity of vibration transducers. As a mechanical device, the principle sensing axis of an accelerometer is not $100 \%$ perpendicular to the mounting axis (surface). This gives rise to the effect that the accelerometer will produce an electrical output even when a mechanical input perpendicular to the principle measurement axis is applied. The quantification of this "defect" parameter is of importance when high accuracy acceleration measurements are performed using accelerometers. This paper gives a brief overview of the system developed by the NMISA to measure the transverse sensitivity of vibration transducers. The paper then explores the validation of the system along with the uncertainty of measurement associated with the calibration system.
\end{abstract}

\section{Section: RESEARCH PAPER}

Keywords: accelerometer; calibration; transverse sensitivity; validation; uncertainty of measurement; ISO 16063-31

Citation: Christiaan S Veldman: Accelerometer transverse sensitivity calibration; validation and uncertainty estimation, Acta IMEKO, vol. 4, no. 2, article 9, June 2015, identifier: IMEKO-ACTA-04 (2015)-02-09

Editor: Paolo Carbone, University of Perugia, Italy

Received September $23^{\text {rd }}, 2014$; In final form February 14 ${ }^{\text {th }}, 2015$; Published June 2015

Copyright: (C) 2015 IMEKO. This is an open-access article distributed under the terms of the Creative Commons Attribution 3.0 License, which permits unrestricted use, distribution, and reproduction in any medium, provided the original author and source are credited

Funding: This work was supported by the Department of Trade and Industry, South Africa

Corresponding author: Ian Veldman, e-mail: CSVeldman@NMISA.org

\section{INTRODUCTION}

Due to their ease of use and low cost, accelerometers are widely considered as the vibration sensor of choice. A variety of different models are required to cover the wide range of vibration measurement applications. To select the accelerometer best suited for a specific application, the user will typically scrutinize the manufacturer's specifications. Apart from the general (usually the most relevant) specifications such as size, sensitivity, frequency- and acceleration ranges, the manufacturer also specifies the relative transverse sensitivity (RTS) of an accelerometer.

For specialized application, the transverse sensitivity is of importance. For some applications, a more accurately known value of the transverse sensitivity might be required [1], [2], [3]. In addition, knowledge of the angle (mechanical orientation) of the transverse sensitivity is also required.

NMISA developed a capability to accurately measure the transverse sensitivity of accelerometers as part of its research in vibration metrology. NMISA modified its existing low frequency accelerometer calibration system to facilitate the measurement of accelerometer transverse sensitivity and will offer this calibration service to industry in the near future.

In section 2, the author defined "transverse sensitivity". The system hardware and specifications are described in section 3. In section 4 , the approach followed to estimate the uncertainty of measurement as well as the major uncertainty of measurement contributors is discussed. The method followed for validating the system, with the validation results are discussed in section 5. Finally, in the concluding section the findings are summarized.

\section{TRANSVERSE SENSITIVITY}

The transverse sensitivity of an accelerometer is defined as the sensitivity to acceleration applied perpendicular to its sensitive axis [4]. The axis of maximum sensitivity of the transducer is not necessarily aligned with the sensitive axis, as shown in Figure 1. As a result, any motion not in line with the sensitive axis will produce an output.

If the transducer is placed in a rectangular co-ordinate system, as shown in Figure 1, the vector, $S_{\max }$, representing the maximum transducer sensitivity can be resolved into two 

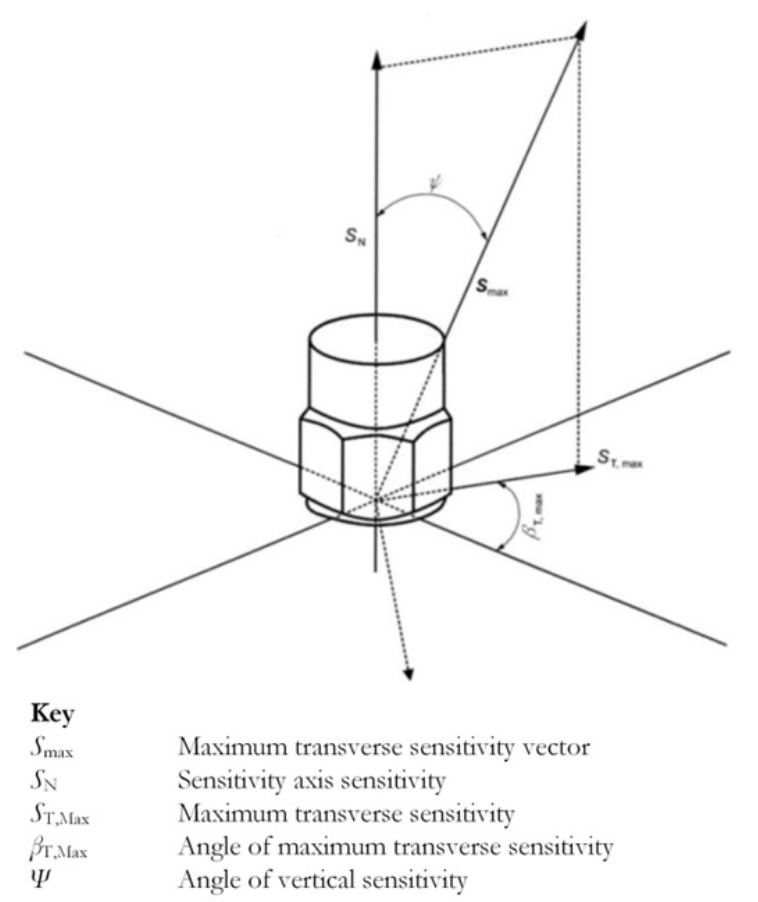

Figure 1. Graphical illustration of transverse sensitivity.

components: the sensitive axis sensitivity, $S_{\mathrm{N}}$, (sensitivity) and the maximum transverse sensitivity, $S_{\mathrm{T} \text { max }}$.

The theoretical transverse sensitivity curve is shown in Figure 2. The transverse sensitivity, expressed as a percentage of the sensitivity, is referred to as the Relative Transverse Sensitivity (RTS). The RTS is dependent on the excitation angle.

For high quality accelerometers, manufacturers supply devices with low RTS, typically $\leq 1 \%$, and with the direction of the lowest transverse sensitivity, $\beta_{\text {TMin }}$, indicated with a red dot on the accelerometer. The manufacturer supplies these low transverse sensitivity devices through selection. That is, they physically measure the transverse sensitivity and select the units

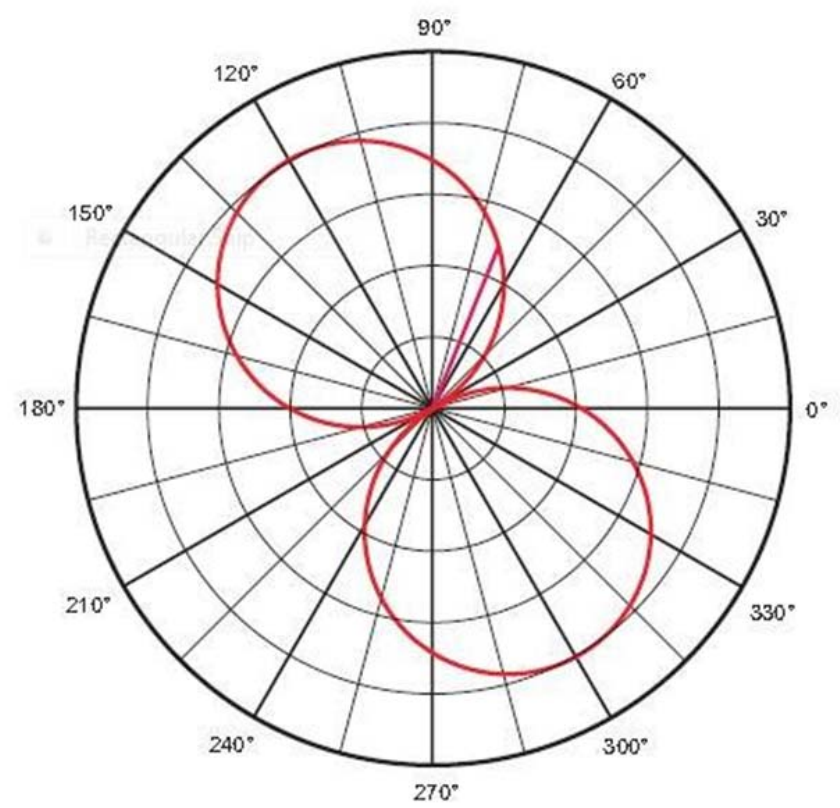

Figure 2. Polar plot indicating a theoretical transverse sensitivity curve for an accelerometer.

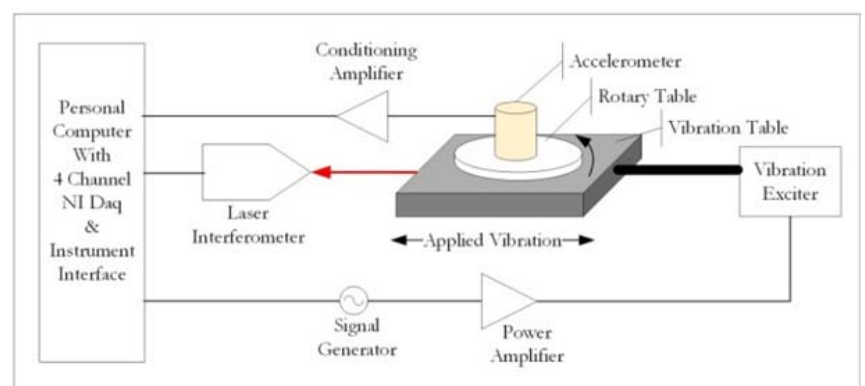

Figure 3. Diagram of the system configuration.

that meet the required RTS specification.

\section{SYSTEM DESCRIPTION}

The transverse sensitivity calibration system of NMISA [5] was developed in compliance with ISO 16063-31 [6]. The transverse sensitivity capability was developed as an extension of the existing primary low frequency accelerometer calibration system. A schematic diagram of the system configuration is shown in Figure 3. The system utilizes the existing long stroke (152 $\mathrm{mm}$ peak to peak) electro-dynamic exciter, connected to an air bearing linear translation stage (ABT). A stepper motor controlled turntable is mounted on top of the ABT (Figure 4). Table 1 provides the system parameters.

For the vibration generator with turntable system implemented by NMISA, once the unit under test (UUT) is mounted on the turntable and all the hardware and cable connections are completed, the in-house developed software is executed.

The software performs a set of procedural steps as part of each transverse sensitivity measurement per turntable angular position as follows:

- Move the turntable to the angular position of interest, (from $0^{\circ}$ to $360^{\circ}$ in $5^{\circ}$ steps);

- Ramp the exciter to the selected vibration level, in frequency and amplitude;

- Sample two analogue inputs simultaneously, streaming the data (time series data) directly to computer storage;

- Apply the three parameter sine fit algorithm (3PSF) [7] to the REF and UUT output voltages time series to calculate acceleration amplitude and the UUT voltage output;

- Calculate the relative transverse sensitivity (RTS) using (1) and (2);

- $\quad$ Record the RTS in the result sheet;

- Plot the RTS on a polar diagram.

Table 1. Parameters of NMISA transverse sensitivity calibration system.

\begin{tabular}{|l|l|}
\hline Vibration frequency range & $5 \mathrm{~Hz}$ to $20 \mathrm{~Hz}$ \\
\hline Transverse acceleration range & $5 \mathrm{~m} / \mathrm{s}^{2}$ to $50 \mathrm{~m} / \mathrm{s}^{2}$ \\
\hline Analogue inputs & $\begin{array}{l}\text { Four simultaneously sampled } 12 \\
\text { bit channels }\end{array}$ \\
\hline Sampling frequency & $500 \mathrm{kHz}$ \\
\hline Turntable rotation angle & $0^{\circ}$ to $360^{\circ}$ \\
\hline Turntable rotation resolution & $1^{\circ}$ \\
\hline Reference & $\begin{array}{l}\text { Polytec OFV-505 Heterodyne } \\
\text { laser interferometer system }\end{array}$ \\
\hline
\end{tabular}




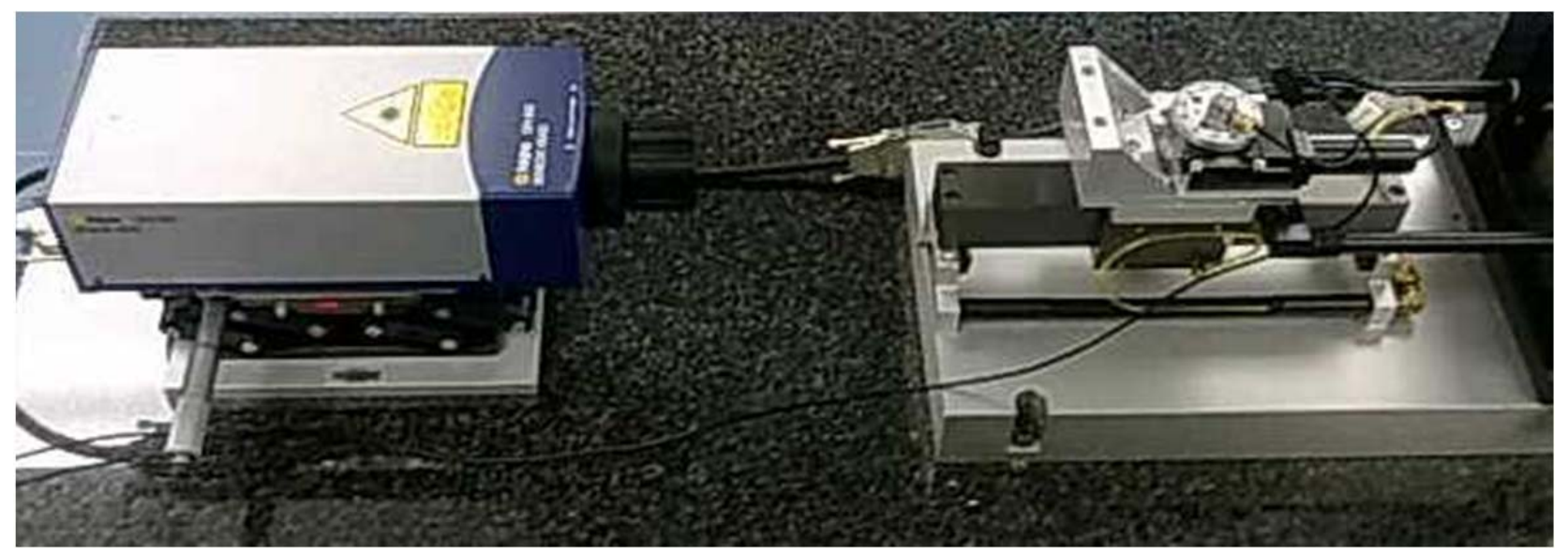

Figure 4. Turntable mounted on top of air-bearing translation stage.

These steps are executed for each angular position from $0^{\circ}$ to $360^{\circ}$ using the selected step size, without the need of any intervention by the metrologist. For the calculation for transverse sensitivity, $S_{\mathrm{T}}$, the following formula was used:

$$
S_{\mathrm{T}}=\frac{\hat{u}_{\text {out }}}{\hat{a}_{\mathrm{T}}}
$$

where $S_{\mathrm{T}}$ is the transverse sensitivity, $\hat{u}_{\text {out }}$ is the amplitude of the output signal of the transducer vibrating perpendicularly to its sensitivity axis, and $\hat{a}_{\mathrm{T}}$ is the acceleration level in the test direction.

The acceleration level is measured by means of a reference transducer and calculated as:

$$
\hat{a}_{\mathrm{T}}=\frac{\hat{u}_{\text {Ref }}}{S_{\text {Ref }}}
$$

where $\hat{\varkappa}_{\text {Ref }}$ is the voltage output of the transducer; and $S_{\text {Ref }}$ is the sensitivity of the reference transducer.

The relative transverse sensitivity, $S_{\mathrm{T}}^{*}$, is calculated from

$S_{\mathrm{T}}^{*}=\frac{S_{\mathrm{T}}}{S_{\mathrm{N}}}$

where $S_{\mathrm{N}}$ is the transducer sensitivity.

Once all the measurements have been completed, the results are saved using an Excel template file. The result sheet also displays the RTS in graphical format, similar to Figure 2.

\section{UNCERTAINTY OF MEASUREMENT}

A conservative approach was followed w.r.t. the consideration of uncertainty contributors. The "worst case" (but still scientifically valid) values were used for the uncertainty contribution. This allows for a single uncertainty calculation that is valid for a wider range of measurement conditions. It also reduces the need to re-calculate the uncertainty budget for each calibration. However, it does not relieve the metrologist of the responsibility of having to consider the uncertainty for each calibration performed. By using the uncertainty values estimated for a specific calibration, instead of the generalized values, an RTS calibration with a smaller uncertainty might be possible.

The uncertainty of measurement (UoM) was estimated in accordance with the GUM [8]. The root uncertainty contributors were identified from the mathematical model in (1), which was expanded to (4) by inserting (1) and (2) into (3):

$$
S_{\mathrm{T}}^{*}=\frac{\mathrm{S}_{\mathrm{Ref}}}{\mathrm{S}_{\mathrm{N}}} \frac{\hat{u}_{\text {out }}}{\hat{u}_{\text {Ref }}}
$$

Through consideration of the mathematical model (4) and the measurement procedure, a detailed set of uncertainty contributors was identified. This full set was reduced to a subset containing the uncertainty contributors with a significant contribution. This process produced the list of "dominant" uncertainty contributors listed in Table 2. A summary of the uncertainty budget is reported in Table 3 .

The voltages for both the reference signal as well as the accelerometer output signal were captured using an A to D converter and applying the 3PSF. The Sine Approximation Method is a well-established and adopted time domain signal processing technique [9]. It requires for the samples to be equidistance sampled, that is that the time between $t_{0}, t_{1}, t_{2} \ldots$ be constant, and if the phase difference between signals is required, that the sampling of the two signals be performed simultaneously. For the system implemented, both these criteria were achieved using a four channel analogue to digital converter (ADC) (four individual channels, not multiplexed channels), with a single sample timing clock, thus synchronising the sampling done by the four ADCs.

It has been established that the 3PSF method is influenced by various factors [10-13] for instance:

- the sampling frequency;

- number of ADC bits;

- number of samples per period to be an integer number;

- number of periods to be a prime number;

- signal to noise ratio (SNR).

Of particular relevance to this application is the SNR [10] as the accelerometer output is a very small signal due to the low transverse sensitivity. As a result, for this application, the SNR is measured. However, for simplicity, a minimum SNR limit of

Table 2. Significant uncertainty contributors

\begin{tabular}{|l|l|}
\hline \multicolumn{1}{|c|}{ Uncertainty Contributor } & \multicolumn{1}{c|}{ Source of Uncertainty } \\
\hline Reference transducer sensitivity & Calibration certificate \\
\hline Acceleration Level & Reference voltage measurement \\
\hline Accelerometer Output Voltage & $\begin{array}{l}\text { Accelerometer voltage } \\
\text { measurement }\end{array}$ \\
\hline Sensitivity (sensitive axis sensitivity) & Calibration certificate \\
\hline Type A Uncertainties & $\begin{array}{l}\text { Statistical means (Standard } \\
\text { Deviation) }\end{array}$ \\
\hline
\end{tabular}


$15 \mathrm{~dB}$ is set for the purpose of calculating the 3PSF uncertainty contribution. For a SNR of $15 \mathrm{~dB}$, the uncertainty in the magnitude determination, $\sigma_{\mathrm{A}}$, is about $0.3 \%$. $\sigma_{\mathrm{A}}$ is calculated using

$$
\sigma_{\mathrm{A}}=\frac{\sqrt{\sigma^{2}+\frac{Q^{2}}{12}}}{\sqrt{\frac{N}{2}}}
$$

where $\sigma_{\mathrm{A}}$ is the 3PSF amplitude precision, $\sigma$ is the zero mean white Gaussian noise, $Q$ is the quantization error $[10,11]$; $N$ is the number of samples.

Due to the inherent small transverse sensitivity of accelerometers, additional steps were required to achieve the SNR limit requirement. This was accomplished by filtering the time signals using a $4^{\text {th }}$ order Butterworth (Infinite Impulse Response (IIR)) digital bandpass filter. The forward-reverse filtering model was applied to accomplish zero phase response. The lower and upper cut-off frequencies were selected to be $0.8909 \cdot f_{\mathrm{v}}$ and $1.225 \cdot f_{\mathrm{v}}$ respectively, where $f_{\mathrm{v}}$ is the vibration frequency.

\subsection{Reference Sensitivity Uncertainty}

For this transverse sensitivity calibration system, the metrologist may choose to use either an accelerometer as the reference device or a laser interferometer (vibrometer). For both options, the sensitivity of the reference device is known from a prior calibration. The uncertainty associated with the sensitivity, along with its coverage factor, is obtained from the calibration of the reference.

\subsection{Acceleration Level Uncertainty}

The voltage amplitude of the reference signal is determined using the 3PSF algorithm [12]. From [13], the uncertainty associated with the amplitude is obtained from (5).

\subsection{Accelerometer Output Voltage Uncertainty}

The uncertainty associated with the accelerometer output voltage is estimated in the same manner as described in section 4.2 since the amplitude is also determined using the 3PSF. However, the calculated uncertainty for this parameter is expected to be larger, due to the smaller SNR, in light of the smaller output voltage.

\subsection{Sensitive Axis Sensitivity}

The uncertainty contribution of the sensitivity will depend on the source of the value of sensitivity. Generally, this sensitivity will be obtained from a valid calibration certificate. In such an instance, the uncertainty will be taken from the certificate. It is possible to determine the sensitivity of the sensitive axis using the calibration system, prior to performing the RTS measurements. In this instance, the uncertainty contribution needs to be calculated separately.

\subsection{Type A Uncertainty}

At each measurement point, $0^{\circ}$ to $355^{\circ}$ in $5^{\circ}$ steps, the system captures an equidistant sampled time series, containing 100 vibration cycles for both the reference- as well as the accelerometer channels. To eliminate the undesired effects introduced by the bandpass filtering, the 3PSF is applied to the centre 50 cycles only. The final voltage amplitude is calculated as the mean and standard deviation of these 50 voltage amplitudes (per channel).

The largest Type A uncertainty was determined by calibrating an accelerometer with a low RTS $(\approx 0.1 \%)$. Using
Table 3. Summary uncertainty budget.

\begin{tabular}{|l|c|c|}
\hline \multicolumn{1}{|c|}{ Source of Uncertainty } & $\begin{array}{c}\text { Estimated } \\
\text { Uncertainty }\end{array}$ & $\begin{array}{c}\text { Probability } \\
\text { Distribution } \\
(\boldsymbol{k})\end{array}$ \\
\hline Reference transducer sensitivity & $0.5 \%$ & 2 \\
\hline $\begin{array}{l}\text { Voltage measurement accuracy } \\
\text { (Sine Fitting) }\end{array}$ & $0.2 \%$ & 1 \\
\hline $\begin{array}{l}\text { Uncertainty in the UUT } \\
\text { sensitivity value }\end{array}$ & $0.8 \%$ & 2 \\
\hline $\begin{array}{l}\text { Voltage measurement accuracy } \\
\text { (Sine Fitting) }\end{array}$ & $0.3 \%$ & 1 \\
\hline Rotation angle accuracy & $0.5^{\circ}$ & $\sqrt{3}$ \\
\hline $\begin{array}{l}\text { Vibration table horizontal } \\
\text { alignment }\end{array}$ & $0.1^{\circ}$ & $\sqrt{3}$ \\
\hline Type A Evaluation & $3.5 \%$ & 1 \\
\hline
\end{tabular}

this accelerometer the largest standard deviation calculated, considering all the measurement points through the complete $360^{\circ}$ rotation was $3.5 \%$. As was to be expected, the Type A uncertainty reached a peak value at an angular position with the lowest RTS.

\section{VALIDATION}

The performance of the transverse sensitivity calibration system and procedure was validated by performing a bilateral interlaboratory comparison (ILC). The ILC partner was the Deutsche Akkreditierungsstelle (DakkS) accredited laboratory, SPEKTRA GmbH, Dresden, Germany.

The purpose of the ILC was to evaluate and validate the metrological operation of the two participating laboratory's accelerometer transverse sensitivity calibration systems and relevant procedures. The differences in the RTS measurements obtained between the two laboratories would support (or disprove) each laboratory's measurement capability, within their stated UoM.

The parameter covered by the ILC was the measurement of the relative transverse sensitivity (RTS) of an accelerometer at $16 \mathrm{~Hz}$. Three accelerometers were used as the ILC transfer devices. The accelerometers that were used are listed in Table 4.

\subsection{Evaluation Criteria}

For each laboratory $i$, the data where $x_{i, s}$, is the maximum relative transverse sensitivity $S_{\mathrm{T}}$, reported and $u_{(x i s)}$, is the reported standard uncertainty associated with the RTS. For each of the comparison artefacts (transfer devices) an ILC reference value $\chi_{\mathrm{R}, S}$ was determined as the weighted mean of the results of $n$ laboratories (for this comparison, $n=2$ ) according to

$$
\begin{gathered}
x_{R, S}=\frac{\sum_{\mathrm{i}=1}^{\mathrm{n}} \frac{\left.\mathrm{x}_{\mathrm{i}, \mathrm{S}} \mathrm{u}_{\mathrm{i}, \mathrm{S}}\right)}{\sum_{i=1}^{n} \frac{1}{u^{2}\left(x_{i, S}\right)}}}{u^{2}\left(x_{R, S}\right)=\frac{1}{\sum_{i=1}^{n} \frac{1}{u^{2}\left(x_{i, S}\right)}}}
\end{gathered}
$$

The degree of equivalence, $D_{\mathrm{LAB}-\mathrm{WM}}$, and $U_{\mathrm{LAB}-\mathrm{WM}}$, was

Table 4. Comparison transfer devices.

\begin{tabular}{|c|l|l|}
\hline No & \multicolumn{1}{|c|}{ Accelerometer } & \multicolumn{1}{c|}{ Serial number } \\
\hline 1 & Endevco 2270M8 & 16194 \\
\hline 2 & PCB 3701G2FA3G & 8353 \\
\hline 3 & PCB J353B01 & 47794 \\
\hline
\end{tabular}


Table 5. Reported ILC Results.

\begin{tabular}{|c|c|c|c|c|}
\hline \multirow{2}{*}{ Accelerometer } & \multicolumn{2}{|c|}{ NMISA } & \multicolumn{2}{c|}{ SPEKTRA } \\
\cline { 2 - 5 } & $\begin{array}{c}\text { RTS } \\
\text { (\%) }\end{array}$ & $\begin{array}{c}\text { Uc } \\
\text { (\%) }\end{array}$ & $\begin{array}{c}\text { RTS } \\
\text { (\%) }\end{array}$ & $\begin{array}{c}\text { Uc } \\
\text { (\%) }\end{array}$ \\
\hline Endevco 2270M8 & 0.8 & 3 & 0.95 & 0.3 \\
\hline PCB 3701G2FA3G & 0.24 & 3 & 0.25 & 0.3 \\
\hline PCB J353B01 & 0.9 & 3 & 0.81 & 0.3 \\
\hline
\end{tabular}

determined for the RTS measurements using

$D_{L a b-W M}=x_{L a b}-x_{W M}$

$U_{L a b-W M}=\sqrt{U_{L a b}^{2}-U_{W M}^{2}}$

where $x_{\text {Lab }}$ represents the measurement results obtained by the laboratory for each RTS and XwM represents the ILC reference value calculated as the weighted mean (WM) using (8). $U_{\text {Lab-WM }}$ is the uncertainty of measurement associated with the calculated $D_{\text {Lab-WM }}$ for $k=2$, calculated using (9).

\subsection{Comparison Results}

The RTSs for the three individual accelerometers as reported by each laboratory (with the associated uncertainties) are reported in Table 5. The calculated comparison reference values (weighted mean values) are reported in Table 6, while the degrees of equivalence (DoE) for each participant is reported in Table 7. The DoE for the measurement results reported by NMISA are shown in graphical format in Figure 5. The graph clearly indicates uncertainties which overlap the reference values indicating NMISA equivalence with SPEKTRA.

\section{CONCLUSIONS}

A transverse sensitivity calibration system was implemented in compliance with ISO 16063-31 by NMISA. The transverse motion is generated using an electro-dynamic vibration exciter with a stepper motor controlled turntable for the angular positioning control.

The requirement for a relatively high $\mathrm{SNR}(\geq 15 \mathrm{~dB})$ was highlighted. This minimum level of SNR is maintained through the use of digital narrow band band-pass filtering.

Four major sources of uncertainty were identified; the

Table 6. Calculated Weighted Mean Values.

\begin{tabular}{|c|c|c|}
\hline \multirow{2}{*}{ Accelerometer } & \multicolumn{2}{|c|}{ Weighted Mean } \\
\cline { 2 - 3 } & RTS $_{\mathrm{WM}}$ & $\boldsymbol{U}_{\mathrm{WM}}$ \\
\cline { 2 - 3 } & $\mathbf{( \% )}$ & $\mathbf{( \% )}$ \\
\hline ENDEVCO 2270M8 & 0.95 & 0.3 \\
\hline PCB 3701G2FA3G & 0.25 & 0.3 \\
\hline PCB J353B01 & 0.81 & 0.3 \\
\hline
\end{tabular}

Table 7. Calculated Degrees of Equivalence.

\begin{tabular}{|c|c|c|c|c|}
\hline \multirow{2}{*}{ Accelerometer } & \multicolumn{4}{|c|}{ Degrees of Equivalence } \\
\cline { 2 - 5 } & D NMISA-WM & $\boldsymbol{U}_{\text {NMISA-WM }}$ & D $_{\text {SPEKTRA-WM }}$ & $\boldsymbol{U}_{\text {SPEKTRA-WM }}$ \\
\cline { 2 - 5 } & $\mathbf{( \% )}$ & $\mathbf{( \% )}$ & $\mathbf{( \% )}$ & $\mathbf{( \% )}$ \\
\hline $\begin{array}{c}\text { ENDEVCO } \\
\text { 2270M8 }\end{array}$ & -0.15 & 3.15 & 0.001 & 0.42 \\
\hline $\begin{array}{c}\text { PCB } \\
\text { 3701G2FA3G }\end{array}$ & -0.01 & 3.01 & 0.0 & 0.42 \\
\hline $\begin{array}{c}\text { PCB } \\
\text { J353B01 }\end{array}$ & 0.09 & 3.11 & -0.001 & 0.42 \\
\hline
\end{tabular}

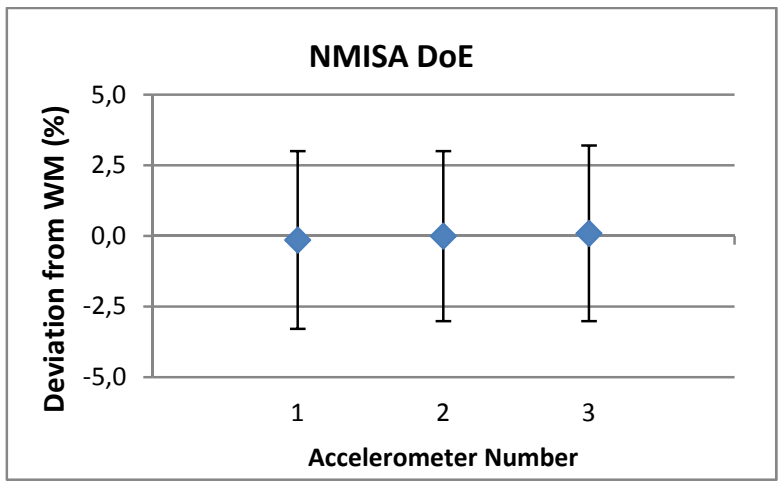

Figure 5. NMISA Degrees of Equivalence.

reference transducer sensitivity, the acceleration level, the accelerometer output voltage and the sensitive axis sensitivity. For this system, the upper limit for the Type A uncertainty was calculated to be $3.5 \%$.

The system was validated through a bi-lateral ILC. The results for the three different accelerometers used, support the UoM estimated by NMISA. The ILC results further indicate that the UoM estimated by NMISA could be considered as fairly conservative.

\section{REFERENCES}

[1] T. Petzsche, "Determination of the transverse sensitivity using a mechanical vibration generator with turntable," ISO TC 108/SC 3/WG 6 Doc. N153, 2007.

[2] J. Dosch and M. Lally, "Automated testing of accelerometer transverse sensitivity," Proceedings of the International Modal Analysis Conference (IMAC), Kissimee, Florida, USA, pp. 1-4, 2003.

[3] R. Sill and E. Seller, "Accelerometer transverse sensitivity measurement using planar orbital motion," Proceedings of the 77th Shock and Vibration Symposium, Monterey, California, USA, pp. 812, November 2006.

[4] ISO, "Mechanical vibration, shock and condition monitoring Vocabulary" ISO 2041, 2009.

[5] C.S. Veldman, "Implementation of an Accelerometer Transverse Sensitivity Measurement System", NCSL International, June 2013.

[6] ISO, "Methods for the calibration of vibration and shock transducers -- Part 31: Testing of transverse vibration sensitivity" ISO 16063-31.

[7] Peter Händle, "Amplitude estimation using IEEE-STD-1057 three-parameter sine wave fit: Statistical distribution, bias and variance", Measurement, vol. 43, pp. 766-770, 2010.

[8] BIPM, IEC, IFCC, ILAC, ISO, IUPAC, IUPAP and OIML 2008 Evaluation of Measurement Data-Guide to the Expression of Uncertainty in Measurement Joint Committee for Guides in Metrology, JCGM 100:2008.

[9] ISO, "Methods for the calibration of vibration and shock transducers -- Part 11: Primary vibration calibration by laser interferometry" ISO 16063-11.

[10] M. Bertocco, C. Narduzi, P. Paglierani, D. Petri, "Accuracy of Effective Bits Estimation Methods", IEEE Instrumentation and Technology Conference, Brussels, Belgium, June 4-6, 1996.

[11] Konrad Heijn, Andrzej Pacut, "Generalized Model of the Quantization Error - A Unified Approach", IEEE Transactions on Instrumentation and Measurement, vol. 34, n. 1, Feb. 1996.

[12] F.Corrêa Alegria, "Bias of amplitude estimation using threeparameter sine fitting in the presence of additive noise", Measurement, 42, pp.748 - 756, 2009.

[13] F. Corrêa Alegria, A. Cruz Serra, "Uncertainty of the Estimates of Sine Wave Fitting of Digital Data in the Presence of Additive Noise", IEEE Instrumentation and Technology Conference, Sorrento, Italy, April 24-27, 2006. 